\title{
Possible observational evidence for cosmic filament spin
}

\author{
Peng Wang $\oplus^{1 凶}$, Noam I. Libeskind $\oplus^{1,2 \bowtie}$, Elmo Tempel ${ }^{3}{ }^{3}$, Xi Kang ${ }^{4,5}$ and Quan Guo ${ }^{6}$
}

\begin{abstract}
Although structures in the Universe form on a wide variety of scales, from small dwarf galaxies to large super clusters, the generation of angular momentum across these scales is poorly understood. Here we investigate the possibility that filaments of galaxies-cylindrical tendrils of matter hundreds of millions of light years across-are themselves spinning. By stacking thousands of filaments together and examining the velocity of galaxies perpendicular to the filament's axis (via their redshift and blueshift), we find that these objects too display vortical motion consistent with rotation, making them the largest objects known to have angular momentum. The strength of the rotation signal is directly dependent on the viewing angle and the dynamical state of the filament. Filament rotation is more clearly detected when viewed edge-on. In addition, the more massive the haloes that sit at either end of the filaments, the more rotation is detected. These results signify that angular momentum can be generated on unexpectedly large scales.
\end{abstract}

$H$ ow angular momentum is generated in a cosmological context is one of the key unsolved problems of cosmology. In the standard model of structure formation, small overdensities present in the early Universe grow via gravitational instability as matter flows from under- to overdense regions. Such a potential flow is irrotational or curl free: there is no primordial rotation in the early Universe and angular momentum must be generated as structures form.

Tidal torque theory $\mathrm{y}^{1-9}$ provides one explanation-the misalignment of the inertia tensor of a gravitationally collapsing region of space with the tidal (shear) field can give rise to torques that spin up the collapsing material ${ }^{1,3,8}$. Such an explanation is valid only in the linear regime, namely in the limit where density perturbations are small with respect to the mean and where flows are laminar. As a collapsing region reaches turnaround, tidal torques cease to be effective and the final angular momentum of a collapsed region is far from what tidal torque theory would predict ${ }^{9-11}$. Although one recent study ${ }^{12}$ has demonstrated that galaxy spin direction (that is, clockwise versus anticlockwise) can be predicted from initial conditions, revealing a critical clue to the nonlinear acquisition of angular momentum, our understanding of spin magnitude, direction and history remains in its infancy. Regions that are still in the linear or quasilinear phase of collapse could provide a better stage for the application of tidal torque theory.

Cosmic filaments ${ }^{13}$, being quasilinear extended topographical features of the galaxy distribution, provide such an environment. Yet, owing to the challenges in characterizing and identifying such objects, potential rotation on the scales of cosmic filaments has been discussed ${ }^{14}$ but never measured until now.

It is known that the cosmic web in general and filaments, in particular, are intimately connected with galaxy formation and evolution $^{15,16}$. They also have a strong effect on galaxy $\operatorname{spin}^{17-22}$, often regulating the direction of how galaxie ${ }^{23-32}$ and their dark matter halos rotate ${ }^{33-41}$. However, it is not known whether the current understanding of structure formation predicts that filaments themselves, being uncollapsed quasilinear objects should spin. A recent study (published on arXiv while this draft was being finalized ${ }^{42}$ examined the velocity field around galactic filaments defined by halo pairs in a large $N$-body simulation and found a statistically significant rotation signal. This is an intriguing finding and, although filaments and their rotation speed are defined differently, the current work in which the observed galaxy distribution is examined in a bid to find possible filament rotation was partly motivated by the theoretical suggestion that filaments may spin ${ }^{42}$.

\section{Results}

After segmenting the galaxy distribution into filaments using a marked point process known as the Bisous model ${ }^{43}$, each filament can be approximated by a rectangle on the sky and thus the galaxies within it may be divided into two regions ( $\mathrm{A}$ and $\mathrm{B}$ ) on either side of the filament spine. The mean redshift difference $\Delta z_{\mathrm{AB}}$ of galaxies between two regions are considered as a proxy for the line-of-sight velocity difference and hence for the filament spin signal. Since measuring mean values among subsamples will by construction result in differences between such averages, any measured value of $\Delta z_{\mathrm{AB}}$ needs to be assigned a significance based on a randomization procedure (explained in Methods). Figure 1 shows the statistical significance of the measured $\Delta z_{\mathrm{AB}}$ as a function of $z_{\mathrm{r} . \mathrm{s} .} / \Delta z_{\mathrm{AB}}$ (where $z_{\text {r.m.s. }}$ is the root mean square (r.m.s.) of the galaxy redshift) - a proxy for the dynamical 'temperature' of the filament (Methods). The number of galaxies in each region is denoted by colour (the effect of galaxy number on this signal is shown in Supplementary Fig. 1). Two salient points can be gleaned here. First, the more galaxies in a given filament, the more inconsistent the redshift difference $\Delta z_{\mathrm{AB}}$ is with random. Second, (as expected) the colder the filament, the more inconsistent the redshift difference is with random. This second point is a generalization-cold filaments with $z_{\text {r.m. }} / \Delta z_{\mathrm{AB}}<1$ and few galaxies can have redshift differences only weakly inconsistent with random expectations. However one may note that as a trend, the colder the filament, the more significant the redshift

'Leibniz-Institut für Astrophysik Potsdam, Potsdam, Germany. ${ }^{2}$ University of Lyon, UCB Lyon 1/CNRS/IN2P3, IPN Lyon (IPNL), Lyon, France. ${ }^{3}$ Tartu Observatory, University of Tartu, Tõravere, Estonia. ${ }^{4}$ Zhejiang University-Purple Mountain Observatory Joint Research Center for Astronomy, Zhejiang University, Hangzhou, China. ${ }^{5}$ Purple Mountain Observatory, Nanjing, China. ${ }^{6}$ Shanghai Astronomical Observatory, Shanghai, China. 凶e-mail: pwang@aip.de; nlibeskind@aip.de 


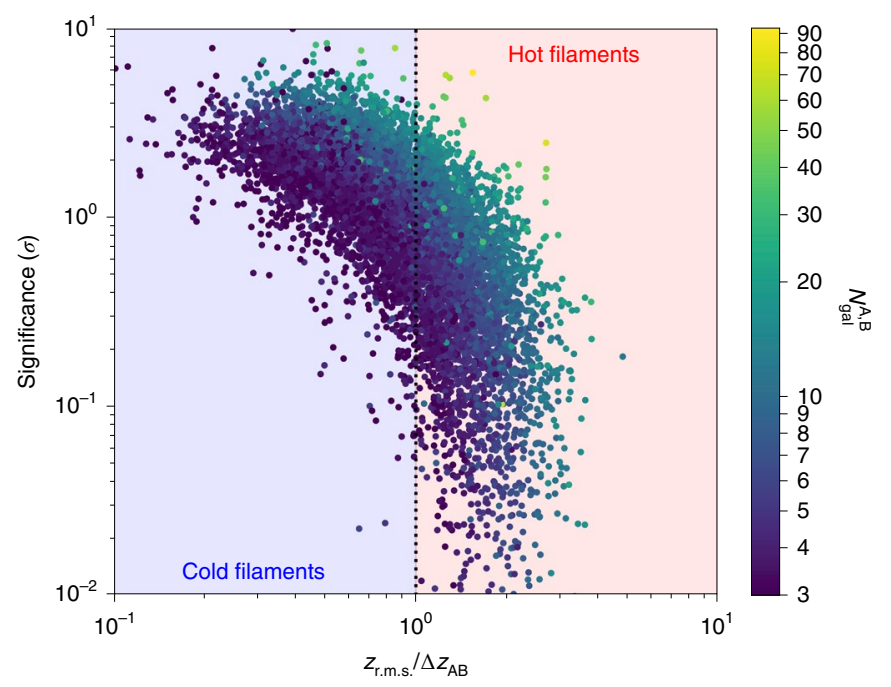

Fig. 1 | The statistical significance of filament rotation. The statistical significance of $\Delta z_{A B}$ being consistent with random is shown as a function of the filament dynamical 'temperature', $z_{\text {r.m.s. }} / \Delta z_{A B}$, in which $\Delta z_{A B}$ is the redshift difference of galaxies between the approaching and receding regions of each filament. The higher this quantity, the more unlikely it is that $\Delta z_{\mathrm{A}}$ is a random occurrence. Each filament is coloured-coded by the number of galaxies it contains, with yellow being rich filaments and purple being poor filaments as designated by the colour bar. At a given value of $z_{\text {r.m.s. }}$ / $\Delta z_{\mathrm{AB}}$, the $\Delta z_{\mathrm{AB}}$ seen in richer filaments is more statistically inconsistent with random. 'Cold' or 'hot' filaments are separated by $z_{\text {r.m.s. }} / \Delta z_{A B}=1$. Note that $N_{\text {gal }}^{A, B}$ is the galaxy number in either region $A$ or region $B$.

difference. In other words, if $\Delta z_{\mathrm{AB}}$ is considered a proxy for filament spin, we observe a spectrum of filaments from dynamically hot that are consistent with random to dynamically cold filaments that are completely inconsistent with random at the many-sigma level. Note that even for dynamically hot filaments, there are a few that are highly inconsistent with random.

The reader will note that the wide distribution of significance seen at a given value of $z_{\text {r.m.s. }} / \Delta z_{\mathrm{AB}}$, is also a reflection of the distribution of inclination angle made by the filament axis with the line of sight. The median value of $z_{\text {r.m.s. }} / \Delta z_{\mathrm{AB}}$ (as well the standard deviation) is shown in Fig. 2 as a function of the angle subtended between the filament spine and the line of sight. Two important points can be observed by this plot: (1) the filament's dynamical temperature $\left(z_{\text {r.m.s. }} / \Delta z_{\mathrm{AB}}\right)$ increases as filaments become parallel to the line of sight. Because the actual value of $z_{\text {r.m.s. }} / \Delta z_{\mathrm{AB}}$ for a given filament is independent of the inclination angle $\phi$, this fact demonstrates that the viewing angle is critical in measuring this quantity and that $z_{\text {r.m.s. }} / \Delta z_{\mathrm{AB}}$ can indeed be used to measure rotation for filaments that are close to perpendicular to the line of sight. Furthermore such a relationship is natural if a significant component of a galaxy's velocity is perpendicular to the filament spine and consistent with rotation or shear. (2) The standard deviation of $z_{\text {r.m.s. }} / \Delta z_{\mathrm{AB}}$ increases as filaments become parallel to the line of sight. This is expected if in addition to rotational motion about the filament spine, galaxies are also travelling along the filament axis-that is, helical motion.

The cumulative distribution of $\Delta z_{\mathrm{A}}$ for both the entire observed filament sample, as well as the randomized trials, is shown in Fig. 3. The cumulative distribution is shown for all filaments (Fig. 3a), filaments whose axis is inclined by $\cos \phi<0.5$ to the line of sight (Fig. $3 \mathrm{~b})$ and dynamically cold filaments $\left(z_{\text {r.m.s. }} / \Delta z_{\mathrm{AB}}<1\right)$ inclined by $\cos \phi<0.2$ (Fig. 3c). The reader will note that even when examining all filaments where the inclination angle is completely ignored (and hence includes filaments viewed along their axis that will probably weaken the signal), the full distribution of $\Delta z_{\mathrm{AB}}$ is inconsistent with randomization tests (see Supplementary Fig. 1 for an examination of the effect of filament inclination angle). This inconsistency increases when considering filaments with $\cos \phi<0.5$ and $\cos \phi<0.2$ that are cold. To quantify the statistical significance of the cumulative distribution one may simply measure, in units of the 10,000 random trial's standard deviation, how far the measured signal is from the mean randomized signal. This is plotted in the top panels of Fig. 3 and shows that randomized trails are statistically inconsistent with the measured signal at very high confidence. In other words, shuffling the redshifts of galaxies in a filament is unlikely to produce redshift differences as great as that observed.

The redshift difference (a proxy for the rotation signal) stacked across various (sub-)samples, is presented in Fig. 4. The following conventions have been adopted. Region A (defined as the region with greater mean redshift) is plotted in the top part of each plot and region $\mathrm{B}$ is plotted in the bottom part. The position of each galaxy along and perpendicular to the filament axis is shown on the $x$ and $y$ axis, respectively, and along the $x$ axis is normalized to the filament's length. Each galaxy is coloured by its redshift difference $\Delta z$, with respect to the mean redshift of all galaxies in the filament according to the colour bar on the right. In the ideal situation where all galaxies exhibit circular or helical motion about the filament axis, such a plot would only have red points in the top part and blue points in the bottom part. The statistical significance of each (sub-)sample, in units of $\sigma$, is indicated on top of each panel.

In Fig. 4a,d,g, we show the stacked rotation signal for all filaments, filaments whose axis subtends an angle $\cos \phi<0.5$ with the line of sight and filaments whose axis subtends an angle $\cos \phi<0.2$ with the line of sight, and that have $z_{\text {r.m.s. }} / \Delta z_{\mathrm{AB}}<1$. Comparing Fig. $4 \mathrm{a}$ with Fig. 4 d, the reader will note what has been mentioned before, namely that merely changing the inclination angle increases the signal. Figure $4 \mathrm{~g}$ shows a very strong rotation signal at $3.3 \sigma-$ when considering dynamically cold filaments that are mostly perpendicular to the line of sight, the rotation signal becomes very convincing.

Since filaments are long tendrils of galaxies often connecting nodes of the cosmic web, the mass of the two halos closest to the filament's two end points are examined for a possible correlation

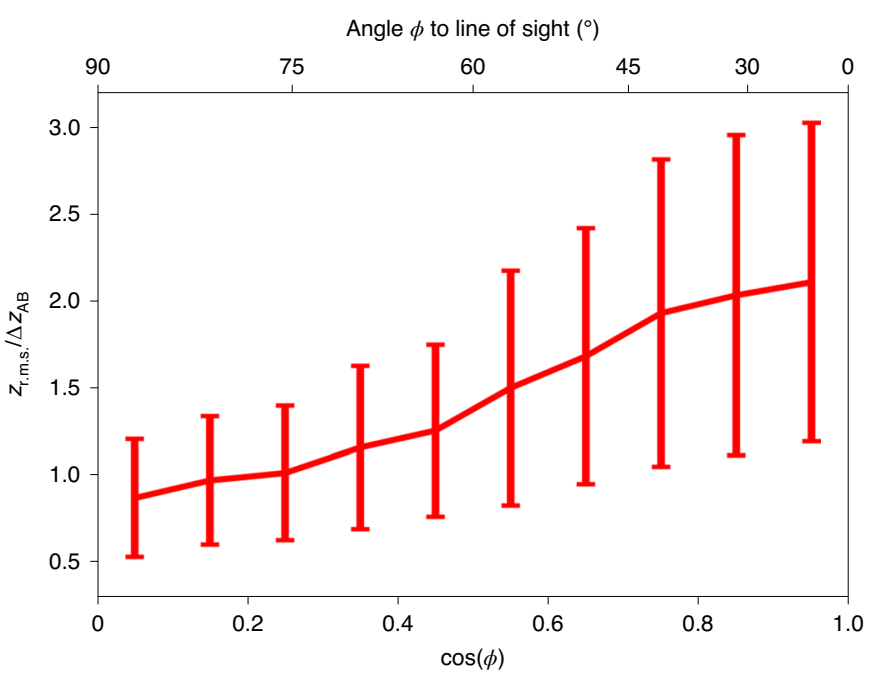

Fig. 2 | The median dynamical 'temperature', $z_{\text {r.m.s. }} / \Delta z_{A B}$, of filaments as a function of the inclination angle $\phi$ between the filament spine and the line of sight. $z_{\text {r.m.s. }}$ is the r.m.s. of galaxy redshift and $\Delta z_{A B}$ is the redshift difference of galaxies on either side of the filament spine. That this quantity increases as the filaments become parallel to the line of sight is consistent with galaxies moving predominantly around the filament spine. Error bars represent the standard deviation about the median. 
a

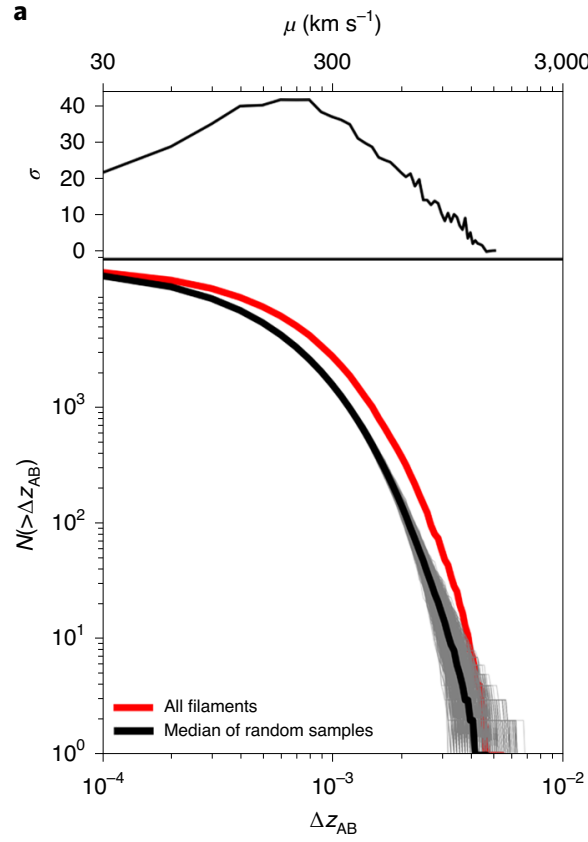

b

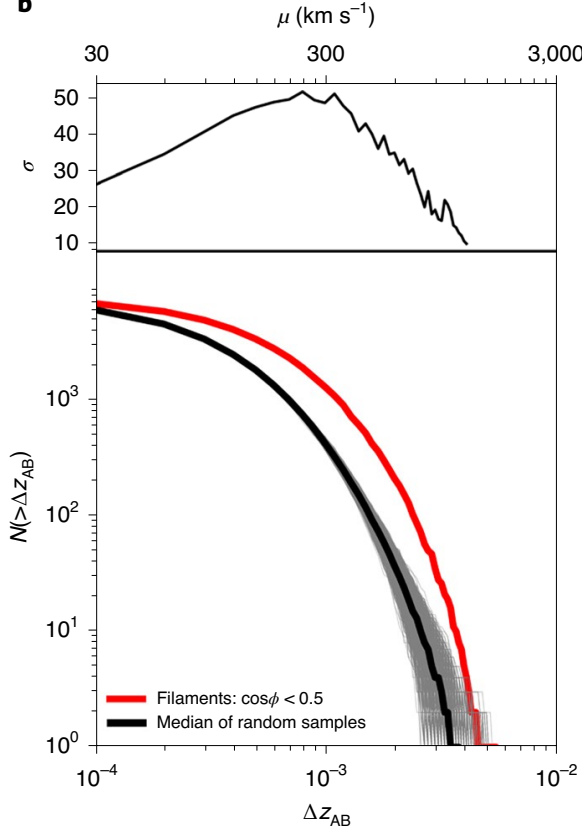

c

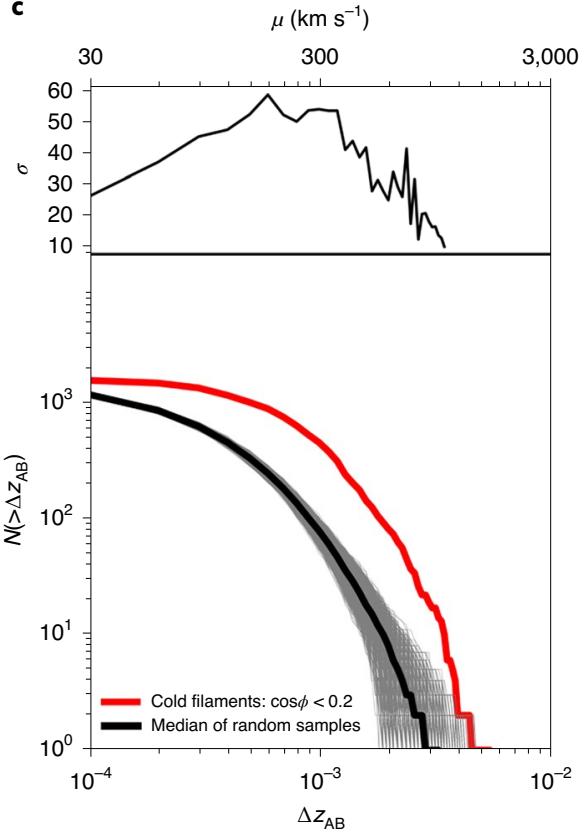

Fig. $3 \mid$ The cumulative distribution of $\Delta z_{A B}$, the redshift difference of galaxies in the approaching and receding regions of each filament. a, All filaments irrespective of viewing angle. $\mathbf{b}$, Filaments whose axis subtends an angle $\cos \phi<0.5$ with the line of sight. $\mathbf{c}$, Filaments whose axis subtends an angle $\cos \phi<0.2$ with the line of sight and that are dynamically cold, namely $z_{\mathrm{r} . \mathrm{m} .} / \Delta z_{\mathrm{AB}}<1$. The red solid line shows the distribution of observed filaments and the 10,000 grey lines indicate the distribution after shuffling the redshifts of galaxies randomly. The median value of these 10,000 random samples is shown as the black solid line. The top panels measure, as a function of $\Delta z_{A}$, the distance, in units of the standard deviation of the randomized distribution, between the measured curve and the mean of the random distributions. The top $x$ axis displays the rotation velocity of the filament in $\mathrm{km} \mathrm{s}^{-1}, \mathrm{calculated}$ as $\mu=c \times \Delta z_{\mathrm{AB}}$.

with the signal strength we measure. We thus perform the following procedure. We search the group catalogue ${ }^{44}$ for the two groups that are closest to the filament's two end points. The reader is referred to that paper for details. In sum, the galaxy groups are detected with a modified friend-of-friend algorithm, where the friend-of-friend groups are divided into subgroups so that each subgroup can be considered as a virialized structure. The mass of each group is estimated using the virial theorem, the size of the group in the sky and the group velocity dispersion along the line of sight. By summing these two group masses, we can ascribe a single mass abutting each filament. We call this mass the 'filament end point mass'.

The three samples of filaments can then be subdivided according to this characteristic. The middle and right columns of Fig. 4 show the stacked signal for the $10 \%$ of the filaments with the smallest filament end point mass (that is, Fig. 4b,e,h) and largest 10\% filament end point mass (Fig. $4 \mathrm{c}, \mathrm{f}, \mathrm{i}$ ) associated halo end points, respectively. The limits of the 10th and 90th percentile group masses of Fig. 4h,i are $\sim 10^{10.4}$ and $\sim 10^{14.3}$, in units of $M_{\odot}$, respectively.

A clear dependence on filament end point mass is seen for all three samples in Fig. 4. The smaller the filament end point mass, the weaker the rotation signal. The filaments with the largest end point mass, most perpendicular to the line of sight, and dynamically cold, show an outstanding $4.2 \sigma$ inconsistency with the null hypothesis of random origin. The fact that the mass of the clusters at the end of each filament directly affects the measured signal is evidence that the signal we are measuring is a physical effect related to gravitational dynamics.

A 'rotation curve' can be constructed for the stacked filament sample by converting the redshift difference into a velocity difference computed as $c \times \Delta z$, where $\Delta z$ is the redshift difference between all the galaxies at given distance and the mean redshift of all galaxies in the filament, and $c$ is the speed of light. This is shown in Fig. 5. We adopt the typical convention that negative (positive) velocities corresponds to approaching (receding) motion. Figure 5 shows that rotation speed increases with distance, reaching a peak of around $\sim 100 \mathrm{~km} \mathrm{~s}^{-1}$ at a distance of $\sim 1 \mathrm{Mpc}$. It then decreases with distance, tending to zero at a distance of greater than $\sim 2 \mathrm{Mpc}$. Incidentally, this behaviour motivates the choice of filament thickness in this study, and should be kept in mind in future studies of the dynamics of cosmic filaments.

\section{Summary and discussion}

The results presented in this paper are consistent with the detection of a signal one would expect if filaments rotated. What is measured and presented here is the redshift difference between two regions on either side of a hypothesized spin axis that is coincident with the filament spine. The full distribution of this quantity (Fig. 3) is inconsistent with random regardless of the viewing angle formed with the line of sight but does strengthen as more perpendicular filaments are examined. It also strengthens when considering dynamically cold filaments-filaments whose galaxies have small r.m.s. values of their redshift. We emphasize that this is not a trivial finding. There is no reason-besides an inherent rotation-that filaments that are dynamically cold should exhibit such a signal.

One may draw an analogy with galaxies here: a disk galaxy viewed edge-on shows a strong $\Delta z_{\mathrm{AB}}$ (consider $\Delta z_{\mathrm{AB}}$ as the Doppler shift from stars on the receding and approaching edge of the galaxy). The value (and significance as measured by the likelihood that it is random) of such a $\Delta z_{\mathrm{A}}$ would decrease as the viewing angle of the galaxy is increased until the galaxy is seen face on. At this point the galaxy would appear 'hot'- the motion of stars along the line of sight being random. In addition, there is a degeneracy between morphology and shape in such situations: a very thin galaxy viewed with a large inclination angle appears ellipsoidal. Since not all galaxies are rotationally supported, some galaxies that appear ellipsoidal will be inclined rotating thin disks while others will be dispersion supported ellipsoids with little inherent rotation. It is this combination of inclination angle and inherent rotation which explains the 

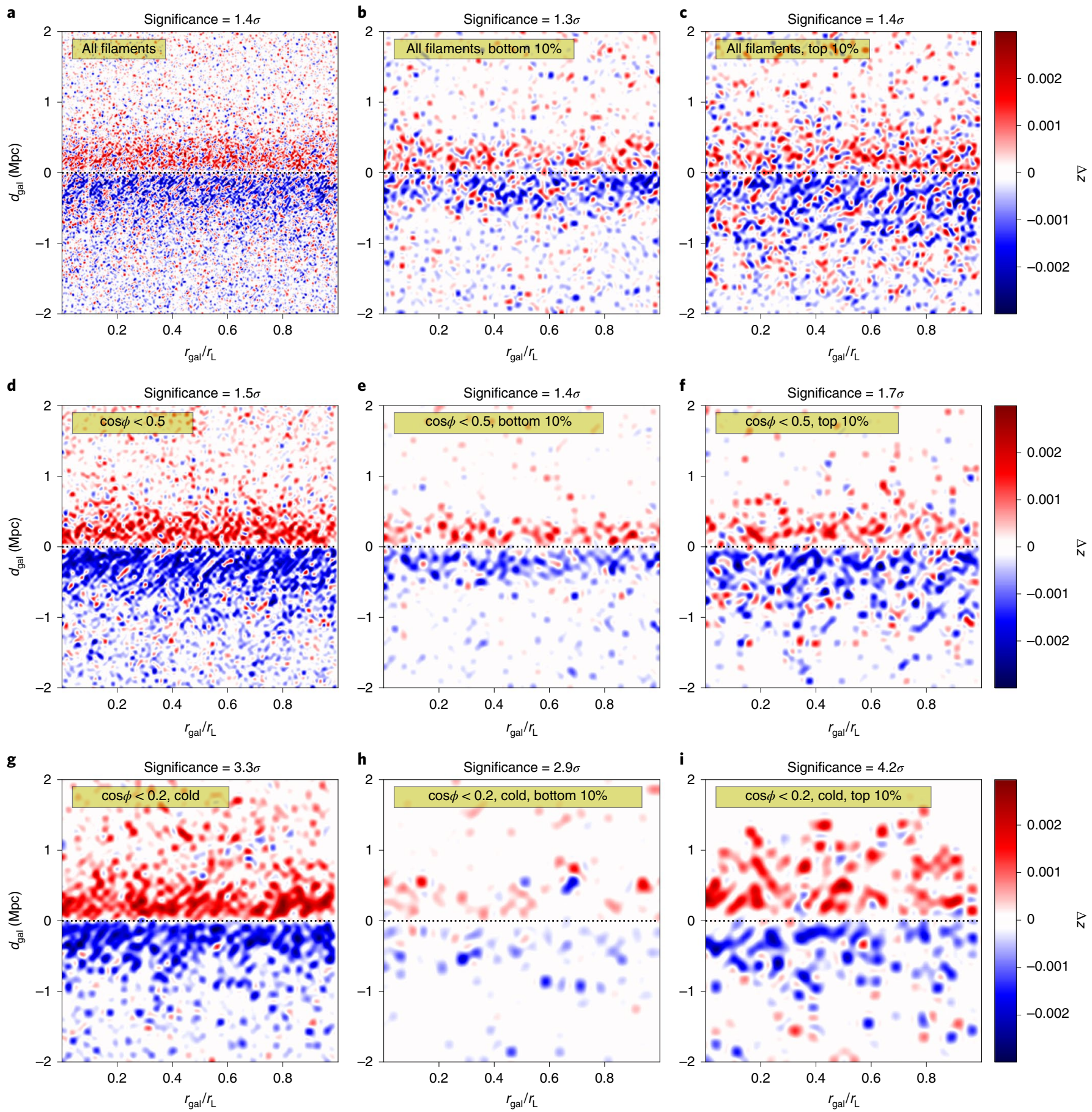

Fig. 4 | The stacked rotation signal of filaments. a-i, Galaxies' position $r_{\text {gal }}$ along the filament are scaled by the length $r_{L}$ of the filament. $d_{g a l}$ is the distance of galaxies to the filament axis. Region A (defined as the region with greater mean redshift) is shown in the top part of each plot and region B is shown in the bottom part. The rows show the stacked rotation signal for all filaments (a-c), filaments whose spine subtends an angle $\cos \phi<0.5$ with the line of sight (d-f), and filaments with $\cos \phi<0.2$ and that have $z_{\text {r.m.s }} / \Delta z_{A B}<1(\mathbf{g}-\mathbf{i})$. The filament sample is divided up according to the group mass at the filaments' two end points: $\mathbf{b}, \mathbf{e}$ and $\mathbf{h}(\mathbf{c}, \mathbf{f}$, and $\mathbf{i})$ show the stacked rotation signal for the $10 \%$ least (largest) filament end point group mass and $\mathbf{a}, \mathbf{d}$ and $\mathbf{g}$ show the signal irrespective of this quantity. The redshift difference is indicated in the colour bar.

spectrum of $z_{\text {r.m.s. }} / \Delta z_{\mathrm{AB}}$ and significance seen in both the galaxy distribution as well as the spin of filaments.

We note that it is the unique combination of small $z_{\text {rm.s. }}$ and large $\Delta z_{\mathrm{AB}}$ that returns a strong statistically significant signal. In other words, there are filaments with large $z_{\text {r.m.s. }}$ and small $\Delta z_{\mathrm{AB}}$ that clearly do not show any signal. This work does not predict that every single filament in the Universe is rotating, rather that there are subsamples-intimately connected to the viewing angle end point mass-that show a clear signal consistent with rotation. This is the main finding of this work. Again, we note that it is not trivial that filaments with low values of $z_{\text {rm.s. }}$ exhibit large values of $\Delta z_{\mathrm{AB}}$. This is only expected if filaments rotate. In Supplementary Fig. 2, we considered other possible biases that could systematically mimic the signal found here, such as the effect of the large-scale motion 


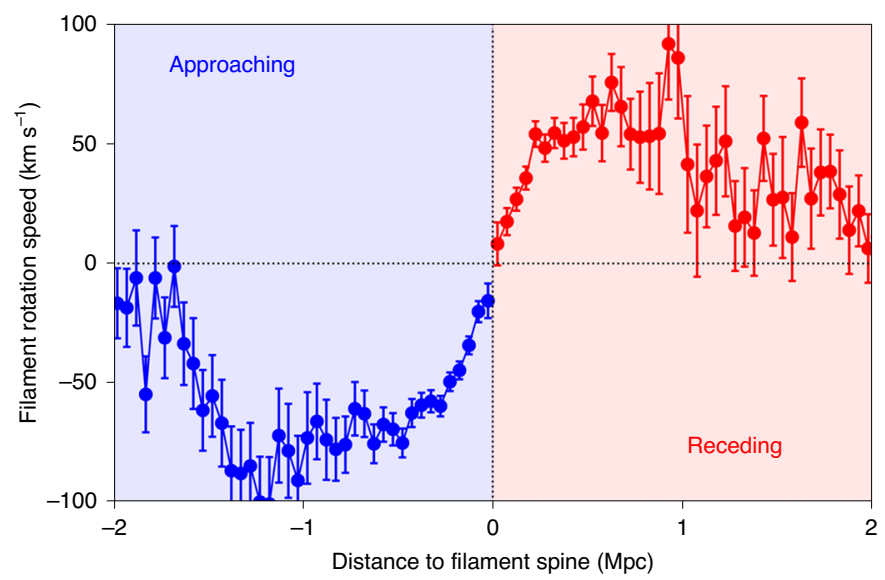

Fig. 5 | The rotation curve of filaments. The filament rotation speed as a function of the distance between galaxies and the filament spine. The rotation speed is calculated by $c \times \Delta z$, where $\Delta z$ is the redshift difference of galaxies at given distance with respect to the redshift of the filament. The distance of galaxies from the filament spine in the receding region is displayed in red and ascribed positive values, while the distance of galaxies in the approaching region is marked in blue and ascribed negative values. Error bars represent the standard deviation about the mean.

of galaxies, finding these are unable to account for the detection found here.

When divided into subsamples based on the mass of the halos that sit at the ends of the filaments, those filaments with the most massive clusters have more significantly spinning signals than either the full sample or the filaments abutting the least massive halos. This gives a hint towards what may be driving the filament spin signal. It is also consistent with the idea that the spin is due to tidal fields influenced by the presence of massive aspherical density perturbations. The existence of a mass dependency is also strong evidence that what we have observed is not a systematic effect due to (for example) cosmological expansion (Supplementary Fig. 2). Any such systematic effects on the measurement of the redshift difference and its association with a velocity difference would not manifest as a mass dependency. The mass dependency opens the door to a number of important questions as to the origin of this rotation or shear, questions that will be examined in future (theoretical) work such as: how is the measured effect engendered by the formation of clusters abutting filaments? Is there an angular momentum correlation between spinning filaments and nearby clusters? And perhaps most importantly: at which stage in structure formation do filaments spin up, and how does this affect the galaxies within them?

According to the Zel'Dovich approximation ${ }^{45}$, the motion of galaxies is accompanied by larger-scale matter flows ${ }^{46,47}$. The mass and velocity flows are usually transported in a 'hierarchical' way: at first, matter collapses along the principal axis of compression forming great cosmic walls. Matter then flows in the wall plane along the direction of the intermediate axis of compression to form filaments. The final stage is the full three-dimensional collapse of an aspherical anisotropic density perturbation wherein matter collapses and flows along the filament axes to form clusters. Under such a model of mass flow, one may expect that filament spin is formed at the second stage from the compression along the intermediate axis of compression. In other words, we expect motion along the filament to still be linear while motion perpendicular to the filament spine to be nonlinear.

The so-called quasilinear regime describes the scales we are examining, where the linear approximation is no longer fully able to describe the dynamics, but the system is not yet fully nonlinear.
'Shell crossing' is generally considered to be the 'moment' where the linear (laminar flow) approximation ceases to accurately describe the dynamics, and nonlinear effects start to dominate. In objects such as the filaments examined here, shell crossing has occurred along the two axes perpendicular to the filament spine but not yet along the filament axis itself. Accordingly, a simple analytical calculation suggests that when a (significant) velocity difference is observed along an axis that has shell-crossed (that is, for filaments that are aligned perpendicular to the line of sight), what is measured is a vortical flow, and filaments can be said to 'spin'. The term 'spin' is a simplification but not an incorrect one. Because a fluid is modelled as a vector field, it is customary to use the vorticity to describe any rotational motion. When filaments are viewed along the axis that has not yet shell-crossed (that is, for filaments that are aligned along the line of sight) any motion is ascribed to a shear that, together with the vorticity, leads to helical motion. In other words, the signal we measure is both consistent with a shear caused by galaxy motion in a sheet, as well as the vortical spin of a filament embedded in that sheet. These two views are not contradictory with each other, since in the picture described above the helicoidal spin of a filament is engendered by the shear of the sheet in which it is embedded combined with vorticity in the directions that have shell-crossed.

We know that from studies of the spatial distributions of smaller galaxies that inhabit the regions between large galaxies that they are accreted in a twofold process: first onto the filament connecting the two larger halos and then second an accretion along the line connecting the halos. In other words, galaxies are first accreted from a direction perpendicular to the filament spine ${ }^{38,48}$ and then along the spine $\mathrm{e}^{38,49,50}$. Assuming filaments can be approximated by a cylindrical potential, such a flow would naturally give rise to an rotation about the filament axis, followed by helical accretion. This is consistent with the theoretical picture formed by ref. ${ }^{42}$, in which the analysis of numerical simulations indicated that the angular momentum of filaments is generated by those particles that collapse along the filament's potential. Taken together, the current study and ref. ${ }^{42}$ demonstrate that angular momentum can be generated on unprecedented scales, opening the door to a new understanding of cosmic spin.

\section{Methods}

The method presented here is fairly straightforward. We begin by using a publicly available catalogue of filaments constructed from the Sloan Digital Sky Survey Data Release 12 galaxy survey ${ }^{51,52}$. Such a catalogue is built using the Bisous algorithm ${ }^{43}$, which identifies curvilinear structures in the galaxy distribution. The Bisous algorithm is not astronomy specific and was developed to identify roads in satellite imaging ${ }^{53-55}$. Each filament is approximated by a cylinder with a given axis that makes a viewing angle $\phi$ with the line of sight. Our hypothesis rests on the assumption that if filaments rotate, then there should be a statistically significant component of a galaxy's velocity that is perpendicular to the filament spine. Furthermore, this component should have opposite signs on either side of the filament spine: one receding and one approaching. To detect such a signal in principle, we would only want to examine filaments whose spine is perpendicular to the line of sight such that a galaxy's redshift (a proxy for the radial component of its velocity) would coincide with its angular velocity. However, restricting the filament sample this way dramatically reduces the sample size. Therefore, we divide the filament catalogue according to viewing angle into three bins: all filaments irrespective of viewing angle, those inclined by more than $60^{\circ}(\cos \phi<0.5)$ with respect to the line of sight, and those dynamically cold (see below) and with a viewing angle more than $\sim 80^{\circ}(\cos \phi<0.2)$. This last sample is chosen for the reason that filaments inclined by more than $\sim 80^{\circ}$ are perpendicular enough that the measured value of $z_{\text {r.m.s. }} / \Delta z_{\mathrm{AB}}$ is probably a good approximation to the inherent one. A consequence of this is that there are more cold filaments with $z_{\text {r.m.s. }} / \Delta z_{\mathrm{AB}}<1$ than hot filaments with $z_{\text {r.m.s. }} / \Delta z_{\mathrm{AB}}>1$ inclined by $\cos \phi<0.2$. In Supplementary Section 1 , the effect of viewing angle is examined in more detail. We find that the filaments whose spines are closer to perpendicular to the line of sight have more significant rotation signals.

Each filament axis delineates two regions on either side of the filament spine. The Bisous filament's have an inherent scale of around $1 \mathrm{Mpc}$. However, all galaxies that are within a $2 \mathrm{Mpc}$ distance from the filament axis are considered to be in one of these two regions. Galaxies farther afield are not considered. We note that most of the galaxies that are within $2 \mathrm{Mpc}$ are within $1 \mathrm{Mpc}$ and that including galaxies 
farther away from the filament spine will 'dilute' the signal as the regions farther from the filament axis will, by construction, include random interlopers.

The mean redshift of galaxies in both regions is calculated. The region with the greater mean redshift is arbitrarily called region $A$ and the region with smaller mean redshift is called region $\mathrm{B}$. The redshift difference $\Delta z_{\mathrm{AB}}=\left\langle z_{\mathrm{A}}\right\rangle-\left\langle z_{\mathrm{B}}\right\rangle$ is computed. The relative speed is then simply $\mu=c \Delta z_{\mathrm{AB}}$, where $c$ is the speed of light. Next we examine whether the filament is dynamically hot or cold by using the redshift $z$ of all galaxies in each filament to compute the r.m.s. namely $z_{\mathrm{rmm}}$ This is then compared with $\Delta z_{\mathrm{A}}$. Dynamically hot filaments have $z_{\text {r.m.s. }}>\Delta z_{\mathrm{A}}$ while cold filaments have r.m.s. values less than the redshift difference. Such a definition precludes the ability to search for a signal consistent with rotation in hot filaments since it is by definition flawed to ascribe a physical significance to a $\Delta z_{\mathrm{AB}}$ if it is less than $z_{\text {r.m.s. }}$ Thus, we do not expect to be able to find a discernible rotation signal in dynamically hot filaments.

A lower limit on the number of galaxies in each region of $\mathrm{A}$ and $\mathrm{B}$ of each filament must be assumed. Without such a limit, we would introduce pathological cases into the analysis, namely filaments with no or just one or two galaxies in region $\mathrm{A}$ or $\mathrm{B}$. Therefore, we only consider filaments with at least three galaxies in each region-it is difficult to justify the computation of an r.m.s. for anything less than this. The three galaxies per region $\mathrm{A}$ and $\mathrm{B}$ is a conservative choice and is the least arbitrary limit that can be applied. Despite this choice, the signal is examined as a function of galaxy number in Supplementary Section 1. In sum: increasing the minimum number of galaxies in each region decreases the sample size and increases the significance of the measured signal.The final fiducial sample consists of 17,181 filaments, and 213,625 galaxies in total.

A statistical significance to each measured value of $\Delta z_{\mathrm{AB}}$ must be obtained to ensure that the measured signal is not simply the result of stochasticity. In other words, we wish to ask the question 'What is the chance that the observed distribution or $\Delta z_{\mathrm{AB}}$ could be reproduced given a random distribution of redshifts (in a given filament)?' To answer this question, the following test is performed. The redshifts of all galaxies in a given filament are randomly shuffled, keeping the position fixed. This is performed 10,000 times per filament. In the poorest filaments with only 6 galaxies (say), there are less than 10,000 unique permutations. However, performing this test 10,000 times, although redundant, will not affect the determination of the significance. For each of these trials, the procedure described above is carried out: namely two regions are defined, their mean redshifts are measured and $\Delta z_{\mathrm{AB}}^{\mathrm{random}}$ is computed. The observed $\Delta z_{\mathrm{A}}$ can then be compared with the distribution of random $\Delta z_{\mathrm{AB}}^{\mathrm{random}}$ on a filament-by-filament basis. The chance that a measured $\Delta z_{\mathrm{A}}$ is consistent with random can then be quantified by where (how many standard deviations from the mean) the measured $\Delta z_{\mathrm{AB}}$ is in the distribution of $\Delta z_{\mathrm{AB}}^{\text {random. }}$

\section{Data availability}

The galaxy and filament catalogues used in current study are published in ref. ${ }^{53}$ and are freely available at the Strasbourg astronomical Data Center (CDS) and at http://cosmodb.to.ee.

\section{Code availability}

The codes used in this study are available from the corresponding authors upon reasonable request.

Received: 30 July 2020; Accepted: 28 April 2021; Published online: 14 June 2021

\section{References}

1. Peebles, P. J. E. Origin of the angular momentum of galaxies. Astrophys. J. 155, 393 (1969).

2. Doroshkevich, A. G. The space structure of perturbations and the origin of rotation of galaxies in the theory of fluctuation. Astrofizika 6, 581-600 (1970)

3. Doroshkevich, A. G. Spatial structure of perturbations and origin of galactic rotation in fluctuation theory. Astrophysics 6, 320-330 (1970).

4. White, S. D. M. Angular momentum growth in protogalaxies. Astrophys. J. 286, 38-41 (1984)

5. Barnes, J. \& Efstathiou, G. Angular momentum from tidal torques. Astrophys J. 319, 575 (1987)

6. van Haarlem, M. \& van de Weygaert, R. Velocity fields and alignments of clusters in gravitational instability scenarios. Astrophys. J. 418, 544 (1993).

7. Bond, J. R. \& Myers, S. T. The peak-patch picture of cosmic catalogs. I. Algorithms. Astrophys. J. Suppl. Ser. 103, 1 (1996).

8. Pichon, C. \& Bernardeau, F. Vorticity generation in large-scale structure caustics. Astron. Astrophys. 343, 663-681 (1999).

9. Porciani, C., Dekel, A. \& Hoffman, Y. Testing tidal-torque theory-I. Spin amplitude and direction. Mon. Not. R. Astron. Soc. 332, 325-338 (2002).

10. Pichon, C. et al. Why do galactic spins flip in the cosmic web? A theory of tidal torques near saddles. Proc. Int. Astron. Union 308, 421-432 (2016).

11. Yu, H.-R. et al. Probing primordial chirality with galaxy spins. Phys. Rev. Lett. 124, 101302 (2020).
12. Motloch, P., Yu, H.-R., Pen, U.-L. \& Xie, Y. An observed correlation between galaxy spins and initial conditions. Nat. Astron. 5, 283-288 (2020).

13. Bond, J. R., Kofman, L. \& Pogosyan, D. How filaments of galaxies are woven into the cosmic web. Nature 380, 603-606 (1996).

14. Neyrinck, M., Aragon-Calvo, M. A., Falck, B., Szalay, A. S. \& Wang, J. Halo spin from primordial inner motions. Open J. Astrophys. 3, 3 (2020).

15. Codis, S. et al. Connecting the cosmic web to the spin of dark haloes: implications for galaxy formation. Mon. Not. R. Astron. Soc. 427, 3320-3336 (2012)

16. Aragon Calvo, M. A., Neyrinck, M. C. \& Silk, J. Galaxy quenching from cosmic web detachment. Open J. Astrophys. 2, 7 (2019).

17. Lee, J. \& Pen, U.-L. Cosmic shear from galaxy spins. Astrophys. J. 532, L5-L8 (2000).

18. Lee, J. \& Erdogdu, P. The alignments of the galaxy spins with the real-space tidal field reconstructed from the 2MASS redshift survey. Astrophys. J. 671, 1248-1255 (2007)

19. Aragón-Calvo, M. A., van de Weygaert, R., Jones, B. J. T. \& van der Hulst, J. M. Spin alignment of dark matter halos in filaments and walls. Astrophys. J. 655, L5-L8 (2007)

20. Libeskind, N. I. et al. The velocity shear tensor: tracer of halo alignment. Mon. Not. R. Astron. Soc. 428, 2489-2499 (2013).

21. Tempel, E., Stoica, R. S. \& Saar, E. Evidence for spin alignment of spiral and elliptical/S0 galaxies in filaments. Mon. Not. R. Astron. Soc. 428, 1827-1836 (2013)

22. Zhang, Y. et al. Spin alignments of spiral galaxies within the large-scale structure from SDSS DR7. Astrophys. J. 798, 17 (2015).

23. Tempel, E. \& Libeskind, N. I. Galaxy spin alignment in filaments and sheets: observational evidence. Astrophys. J. 775, L42 (2013).

24. Dubois, Y. et al. Dancing in the dark: galactic properties trace spin swings along the cosmic web. Mon. Not. R. Astron. Soc. 444, 1453-1468 (2014).

25. Forero-Romero, J. E., Contreras, S. \& Padilla, N. Cosmic web alignments with the shape, angular momentum and peculiar velocities of dark matter haloes. Mon. Not. R. Astron. Soc. 443, 1090-1102 (2014).

26. Codis, S., Pichon, C. \& Pogosyan, D. Spin alignments within the cosmic web: a theory of constrained tidal torques near filaments. Mon. Not. R. Astron. Soc. 452, 3369-3393 (2015).

27. Pahwa, I. et al. The alignment of galaxy spin with the shear field in observations. Mon. Not. R. Astron. Soc. 457, 695-703 (2016).

28. Wang, P., Guo, Q., Kang, X. \& Libeskind, N. I. The spin alignment of galaxies with the large-scale tidal field in hydrodynamic simulations. Astrophys. J. 866, 138 (2018).

29. Codis, S. et al. Galaxy orientation with the cosmic web across cosmic time. Mon. Not. R. Astron. Soc. 481, 4753-4774 (2018).

30. Krolewski, A. et al. Alignment between filaments and galaxy spins from the MaNGA integral-field survey. Astrophys. J. 876, 52 (2019).

31. Welker, C. et al. The SAMI galaxy survey: first detection of a transition in spin orientation with respect to cosmic filaments in the stellar kinematics of galaxies. Mon. Not. R. Astron. Soc. 491, 2864-2884 (2020).

32. Kraljic, K., Davé, R. \& Pichon, C. And yet it flips: connecting galactic spin and the cosmic web. Mon. Not. R. Astron. Soc. 493, 362-381 (2020).

33. Hahn, O., Porciani, C., Carollo, C. M. \& Dekel, A. Properties of dark matter haloes in clusters, filaments, sheets and voids. Mon. Not. R. Astron. Soc. 375, 489-499 (2007)

34. Hahn, O., Carollo, C. M., Porciani, C. \& Dekel, A. The evolution of dark matter halo properties in clusters, filaments, sheets and voids. Mon. Not. R. Astron. Soc. 381, 41-51 (2007).

35. Trowland, H. E., Lewis, G. F. \& Bland-Hawthorn, J. The cosmic history of the spin of dark matter halos within the large-scale structure. Astrophys. J. 762 $72(2013)$

36. Laigle, C. et al. Swirling around filaments: are large-scale structure vortices spinning up dark haloes? Mon. Not. R. Astron. Soc. 446, 2744-2759 (2015)

37. Wang, P. \& Kang, X. A general explanation on the correlation of dark matter halo spin with the large-scale environment. Mon. Not. R. Astron. Soc. 468, L123-L127 (2017).

38. Wang, P. \& Kang, X. The build up of the correlation between halo spin and the large-scale structure. Mon. Not. R. Astron. Soc. 473, 1562-1569 (2018).

39. Lee, J. Revisiting the galaxy shape and spin alignments with the large-scale tidal field: an effective practical model. Astrophys. J. 872, 37 (2019).

40. Ganeshaiah Veena, P. et al. The cosmic ballet: spin and shape alignments of haloes in the cosmic web. Mon. Not. R. Astron. Soc. 481, 414-438 (2018).

41. Ganeshaiah Veena, P., Cautun, M., Tempel, E., van de Weygaert, R. \& Frenk, C. S. The cosmic ballet II: spin alignment of galaxies and haloes with large-scale filaments in the EAGLE simulation. Mon. Not. R. Astron. Soc. 487, 1607-1625 (2019).

42. Xia, Q., Neyrinck, M. C., Cai, Y.-C. \& Aragón-Calvo, M. A. Intergalactic filaments spin. Preprint at https://arxiv.org/abs/2006.02418 (2020).

43. Stoica, R. S., Martínez, V. J. \& Saar, E. A three-dimensional object point process for detection of cosmic filaments. J. R. Stat. Soc. Ser. C 56, 459-477 (2007). 
44. Tempel, E., Tuvikene, T., Kipper, R. \& Libeskind, N. I. Merging groups and clusters of galaxies from the SDSS data. The catalogue of groups and potentially merging systems. Astron. Astrophys. 602, A100 (2017).

45. Zel'Dovich, Y. B. Gravitational instability: an approximate theory for large density perturbations. Astron. Astrophys. 500, 13-18 (1970).

46. Icke, V. \& van de Weygaert, R. The galaxy distribution as a Voronoi foam. Q. J. R. Astron. Soc. 32, 85-112 (1991).

47. Cautun, M., van de Weygaert, R., Jones, B. J. T. \& Frenk, C. S. Evolution of the cosmic web. Mon. Not. R. Astron. Soc. 441, 2923-2973 (2014).

48. Kang, X. \& Wang, P. The accretion of dark matter subhalos within the cosmic web: primordial anisotropic distribution and its universality. Astrophys. J. 813, 6 (2015).

49. Libeskind, N. I., Knebe, A., Hoffman, Y. \& Gottlöber, S. The universal nature of subhalo accretion. Mon. Not. R. Astron. Soc. 443, 1274-1280 (2014).

50. Shi, J., Wang, H. \& Mo, H. J. Flow patterns around dark matter halos: the link between halo dynamical properties and large-scale tidal field. Astrophys. J. 807, 37 (2015).

51. Eisenstein, D. J. et al. SDSS-III: massive spectroscopic surveys of the distant Universe, the Milky Way, and extra-solar planetary systems. Astron. J. 142, 72 (2011).

52. Alam, S. et al. The eleventh and twelfth data releases of the Sloan Digital Sky Survey: final data from SDSS-III. Astrophys. J. Suppl. Ser. 219, 12 (2015).

53. Tempel, E. et al. Detecting filamentary pattern in the cosmic web: a catalogue of filaments for the SDSS. Mon. Not. R. Astron. Soc. 438, 3465-3482 (2014).

54. Tempel, E., Stoica, R. S., Kipper, R. \& Saar, E. Bisous model-detecting filamentary patterns in point processes. Astron. Comput. 16, 17-25 (2016).

55. Libeskind, N. I. et al. Tracing the cosmic web. Mon. Not. R. Astron. Soc. 473, 1195-1217 (2018).

\section{Acknowledgements}

We acknowledge stimulating discussions with M. S. Pawlowski, S. Gottlöeber, Y. Hoffman and Z.-z. Li. We acknowledge R. Kipper for a double-blind examination at the beginning of this project. P.W. especially acknowledges his wife N. Wang and daughter T.-e. Wang for their understanding and their help of his home office during the COVID-19 pandemic. P.W., N.I.L., X.K. and Q.G. acknowledge support from the joint Sino-German DFG research Project 'The Cosmic Web and its impact on galaxy formation and alignment' (DFG-LI 2015/5-1, NSFC no. 11861131006). N.I.L. acknowledges financial support of the Project IDEXLYON at the University of Lyon under the Investments for the Future Program (ANR-16-IDEX-0005). E.T. was supported by ETAg grants IUT40-2, PRG1006 and by the EU through the ERDF CoE TK133. X.K. acknowledges financial support by the NSFC (no. 11825303, 11333008), the 973 programme (no. 2015CB857003), and was also partially supported by the China Manned Space Program through its Space Application System. Q.G. acknowledges financial support of Shanghai Pujiang Program (no. 19PJ1410700). This work is partially supported by the cosmology simulation database (CSD) in the National Basic Science Data Center (NBSDC-DB-10, no. 2020000088).

\section{Author contributions}

P.W. carried out all measurements and quantitative analysis, and contributed to the writing of the methods and results. N.I.L. conceived the idea of the project and led the writing. E.T. contributed observational data, the filament catalogue and essential discussions. X.K. and Q.G. contributed significantly to the overall science interpretation and essential discussions. All co-authors contributed by their varied contributions to the science interpretation. All co-authors contributed to the commenting on this manuscript as part of an internal review process.

\section{Competing interests}

The authors declare no competing interests.

\section{Additional information}

Supplementary information The online version contains supplementary material available at https://doi.org/10.1038/s41550-021-01380-6.

Correspondence and requests for materials should be addressed to P.W. or N.I.L. Peer review information Nature Astronomy thanks Joss Bland-Hawthorn, Nelson Padilla and the other, anonymous, reviewer(s) for their contribution to the peer review of this work. Reprints and permissions information is available at www.nature.com/reprints. Publisher's note Springer Nature remains neutral with regard to jurisdictional claims in published maps and institutional affiliations.

(C) The Author(s), under exclusive licence to Springer Nature Limited 2021, corrected publication 2021 